Article

\title{
A Fixed Point Approach to the Stability of a Mean Value Type Functional Equation
}

\author{
Soon-Mo Jung ${ }^{1, *}$ (i) and Yang-Hi Lee ${ }^{2}$ \\ 1 Mathematics Section, Hongik University, Sejong 30016, Korea \\ 2 Department of Mathematics Education, Gongju National University of Education, Gongju 32553, Korea; \\ yanghi2@hanmail.net \\ * Correspondence: smjung@hongik.ac.kr
}

Received: 3 November 2017; Accepted: 5 December 2017; Published: 13 December 2017

Abstract: We prove the generalized Hyers-Ulam stability of a mean value type functional equation $f(x)-g(y)=(x-y) h(x+y)$ by applying a method originated from fixed point theory.

Keywords: Hyers-Ulam stability; mean value type functional equation; fixed point method; fixed point

\section{Introduction}

Ulam [1] proposed the stability problem of functional equations:

"Suppose $G_{1}$ is a group and $G_{2}$ is a metric group with the metric $d(\cdot, \cdot)$. Given $\varepsilon>0$, does there exist a $\delta>0$ such that if a function $h: G_{1} \rightarrow G_{2}$ satisfies inequality $d(h(x y), h(x) h(y))<\delta$ for all $x, y \in G_{1}$, then there exists a homomorphism $H: G_{1} \rightarrow G_{2}$ with $d(h(x), H(x))<\varepsilon$ for all $x \in G_{1}$ ?"

The Ulam's problem for the Cauchy additive equation

$$
f(x+y)=f(x)+f(y)
$$

was solved by Hyers when $G_{1}$ and $G_{2}$ are Banach spaces. Indeed, Hyers [2] proved that every solution to the inequality $\|f(x+y)-f(x)-f(y)\| \leq \varepsilon$ (for all $x, y$ ) can be approximated by an additive function. In that case, the Cauchy additive equation is said to satisfy the Hyers-Ulam stability. This terminology is also applied to the case of other functional equations. Thereafter, a number of mathematicians tackled this problem (cf. [3-5]). For a more detailed description of such terminology, we refer the reader to [2,6-9].

The functional equation

$$
f(x)-g(y)=(x-y) h(x+y),
$$

where $x, y \in \mathbb{R}$ (the set of all real numbers), arises from the mean value theory and it characterizes polynomials of degree one or two. This functional equation was first studied by Aczél in 1963 and also independently by Haruki (see $[10,11])$. The functional Equation (2) was generalized by Kannappan, Sahoo and Jacobson [12] (see also [13] (Theorem 2.5) or [10,11]).

We summarize a result of Aczél concerning the solution to (2) in the following theorem.

Theorem 1. Suppose $X$ is a field of characteristic different from 2. The functions $f, g, h: X \rightarrow X$ satisfy the functional Equation (2) for all $x, y \in X$ if and only if there exist $a, b, c \in X$ such that

$$
f(x)=g(x)=a x^{2}+b x+c \text { and } h(x)=a x+b
$$


for all $x \in X$.

In this paper, we investigate the generalized Hyers-Ulam stability of the mean value type functional Equation (2) by using a method originated from fixed point theory in the sense of Cădariu and Radu (see [14-16]). On one hand, this paper is a continuation of the previous work [17]. On the other hand, the present paper is a good complement to previous work because we make use of the fixed point method in this paper while we used the direct method in [17].

\section{Main Results}

Fixed point theory has a variety of applications, e.g., it has an application in the split feasibility problem for modeling inverse problems or in signal processing (see [18]).

First, a theorem of Margolis and Diaz will be introduced. As usual, we will denote by $\mathbb{N}_{0}$ the set of all nonnegative integers, i.e., $\mathbb{N}_{0}=\mathbb{N} \cup\{0\}$.

Theorem 2. ([19] or [20]) Suppose that $(X, d)$ is a complete generalized metric space, where the metric $d$ may assume an infinite value, and that $J: X \rightarrow X$ is a strictly contractive mapping with Lipschitz constant $0<L<1$. Then, for any element $x \in X$, either $d\left(J^{n} x, J^{n+1} x\right)=+\infty$ for all $n \in \mathbb{N}_{0}$ or there exists a $k \in \mathbb{N}_{0}$ such that

(i) $d\left(J^{n} x, J^{n+1} x\right)<+\infty$ for all $n \geq k$;

(ii) the sequence $\left\{J^{n} x\right\}$ is convergent to a fixed point $y^{*}$ of $J$;

(iii) $y^{*}$ is the unique fixed point of $J$ in $Y:=\left\{y \in X: d\left(J^{k} x, y\right)<+\infty\right\}$;

(iv) $d\left(y, y^{*}\right) \leq \frac{1}{1-L} d(y, J y)$ for all $y \in Y$.

In the present paper, assume that $X$ is a commutative unital Banach algebra over $\mathbb{K}$ (or $X$ is a normed field of characteristic different from 2), where $\mathbb{K}$ denotes either $\mathbb{R}$ or $\mathbb{C}$ (see [21] (Chapter 10) for an exact definition of unital Banach algebra and we note that $X$ has a unit element $e$ with $\|e\|=1$ ).

For any given mappings $f, g, h: X \rightarrow X$, we will use the following notation

$$
D(x, y):=f(x)-g(y)-(x-y) h(x+y)
$$

for $x, y \in X$.

Theorem 3. Assume that $X$ is a commutative unital Banach algebra over $\mathbb{K}$ with a unit element e (or $X$ is a normed field of characteristic different from 2). Suppose a function $\varphi: X \times X \rightarrow[0, \infty)$ satisfies either

$$
\varphi(2 x, 2 y) \leq 4 L \varphi(x, y) \quad(\text { for all } x, y \in X \backslash\{0\})
$$

or

$$
4 \varphi\left(\frac{x}{2}, \frac{y}{2}\right) \leq L \varphi(x, y) \quad(\text { for all } x, y \in X \backslash\{0\}),
$$

where $L$ is a real constant with $0<L<1$. If functions $f, g, h: X \rightarrow X$ satisfy the inequality

$$
\|f(x)-g(y)-(x-y) h(x+y)\| \leq \varphi(x, y)
$$

for all $x, y \in X$, then there exists a unique $\mathbb{K}$-linear mapping $H: X \rightarrow X$ such that

$$
\begin{aligned}
& \|f(x)-x(H(x)+h(0))-f(0)\| \\
& \leq \begin{cases}\varphi(x, 0)+\varphi(0,0)+\frac{\Phi(x,\|x\| e)}{1-L} & \text { if } \varphi \text { satisfies (4), } \\
\varphi(x, 0)+\varphi(0,0)+\frac{L \Phi(x,\|x\| e)}{1-L} & \text { if } \varphi \text { satisfies (5) }\end{cases}
\end{aligned}
$$




$$
\begin{gathered}
\|g(x)-x(H(x)+h(0))-g(0)\| \\
\leq\left\{\begin{aligned}
\varphi(0, x)+\varphi(0,0)+\frac{\Phi(x,\|x\| e)}{1-L} & \text { if } \varphi \text { satisfies (4), } \\
\varphi(0, x)+\varphi(0,0)+\frac{L \Phi(x,\|x\| e)}{1-L} & \text { if } \varphi \text { satisfies (5) }
\end{aligned}\right. \\
\|h(x)-H(x)-h(0)\| \leq \begin{cases}\frac{\Phi(x,\|x\| e)}{(1-L)\|x\|} & \text { if } \varphi \text { satisfies (4), } \\
\frac{L \Phi(x,\|x\| e)}{(1-L)\|x\|} & \text { if } \varphi \text { satisfies (5) }\end{cases} \\
H(x)=H(e) x
\end{gathered}
$$

for all $x \in X \backslash\{-e, 0, e\}$, where

$$
\begin{aligned}
\Phi(x,\|x\| e):= & \varphi\left(\frac{x+\|x\| e}{2}, \frac{x-\|x\| e}{2}\right)+\varphi\left(\frac{x+\|x\| e}{2}, \frac{\|x\| e-x}{2}\right) \\
& +\varphi\left(\frac{x-\|x\| e}{2}, \frac{\|x\| e-x}{2}\right)+\varphi\left(\frac{x-\|x\| e}{2}, \frac{x-\|x\| e}{2}\right) \\
& +\frac{1}{2} \varphi\left(x+\frac{\|x\| e}{2}, x-\frac{\|x\| e}{2}\right)+\frac{1}{2} \varphi\left(x+\frac{\|x\| e}{2}, \frac{\|x\| e}{2}-x\right) \\
& +\frac{1}{2} \varphi\left(x-\frac{\|x\| e}{2}, \frac{\|x\| e}{2}-x\right)+\frac{1}{2} \varphi\left(x-\frac{\|x\| e}{2}, x-\frac{\|x\| e}{2}\right)
\end{aligned}
$$

for any $x \in X \backslash\{-e, 0, e\}$.

Proof. We denote by $S$ the set of all functions $u: X \rightarrow X$ with $u(0)=0$. A generalized metric $d$ on $S$ is defined by

$$
d(u, v):=\inf \left\{K>0:\|u(x)-v(x)\| \leq K \frac{\Phi(x,\|x\| e)}{\|x\|} \text { for all } x \in X \backslash\{-e, 0, e\}\right\}
$$

for all $u, v \in S$. Then it is easy to show that $(S, d)$ is a complete generalized metric space. Hence, we omit its proof. The proof of this theorem will be divided into two cases: One is the case when $\varphi$ satisfies (4) and the other is that $\varphi$ satisfies (5).

Case 1. Assume that $\varphi$ satisfies (4). Let us define a mapping $J: S \rightarrow S$ by

$$
J u(x):=\frac{1}{2} u(2 x) \quad(x \in X)
$$

for all $u \in S$. Notice that

$$
J^{n} u(x)=\frac{1}{2^{n}} u\left(2^{n} x\right)
$$

for all $n \in \mathbb{N}_{0}$ and $x \in X$.

From (4) and (10), it follows that

$$
\Phi(2 x,\|2 x\| e) \leq 4 L \Phi(x,\|x\| e)
$$

for all $x \in X \backslash\{-e, 0, e\}$. Assume that $u, v \in S$ and $K \in[0, \infty]$ is an arbitrary constant with $d(u, v) \leq K$. By definition of $d$, we have

$$
\|J u(x)-J v(x)\|=\frac{1}{2}\|u(2 x)-v(2 x)\| \leq \frac{K}{2} \frac{\Phi(2 x,\|2 x\| e)}{\|2 x\|} \leq L K \frac{\Phi(x,\|x\| e)}{\|x\|}
$$

for all $x \in X \backslash\{-e, 0, e\}$, which yields that

$$
d(J u, J v) \leq L d(u, v)
$$


for any $u, v \in S$. This implies that $J$ is a strictly contractive self-mapping of $S$ with the Lipschitz constant $L$.

Let us define a mapping $h^{\prime}: X \rightarrow X$ by $h^{\prime}(x):=h(x)-h(0)$. By a long calculation and (3), we get

$$
\begin{aligned}
h^{\prime}(x)-J h^{\prime}(x) & h(x)-h(0)-\frac{h(2 x)-h(0)}{2} \\
=\frac{1}{\|x\|}(- & D\left(\frac{x+\|x\| e}{2}, \frac{x-\|x\| e}{2}\right)+D\left(\frac{x+\|x\| e}{2}, \frac{\|x\| e-x}{2}\right) \\
& -D\left(\frac{x-\|x\| e}{2}, \frac{\|x\| e-x}{2}\right)+D\left(\frac{x-\|x\| e}{2}, \frac{x-\|x\| e}{2}\right) \\
& +\frac{1}{2} D\left(x+\frac{\|x\| e}{2}, x-\frac{\|x\| e}{2}\right)-\frac{1}{2} D\left(x+\frac{\|x\| e}{2}, \frac{\|x\| e}{2}-x\right) \\
+ & \left.\frac{1}{2} D\left(x-\frac{\|x\| e}{2}, \frac{\|x\| e}{2}-x\right)-\frac{1}{2} D\left(x-\frac{\|x\| e}{2}, x-\frac{\|x\| e}{2}\right)\right)
\end{aligned}
$$

for all $x \in X \backslash\{-e, 0, e\}$. By (6), (10) and from the last equality, it follows that

$$
\left\|h^{\prime}(x)-J h^{\prime}(x)\right\|=\left\|h(x)-h(0)-\frac{h(2 x)-h(0)}{2}\right\| \leq \frac{\Phi(x,\|x\| e)}{\|x\|}
$$

for all $x \in X \backslash\{-e, 0, e\}$, which means that $d\left(h^{\prime}, J h^{\prime}\right) \leq 1<\infty$ by definition of $d$.

According to Theorem 2, the sequence $\left\{J^{n} h^{\prime}\right\}$ converges to a fixed point $H: X \rightarrow X$ of $J$ which is unique in the set $T=\left\{u \in S: d\left(h^{\prime}, u\right)<\infty\right\}$. We note that

$$
d\left(h^{\prime}, H\right) \leq \frac{1}{1-L} d\left(h^{\prime}, J h^{\prime}\right) \leq \frac{1}{1-L},
$$

from which the first inequality in (9) follows.

By (3) and a routine calculation, we obtain

$$
\begin{aligned}
& x J^{n} h^{\prime}(e)-J^{n} h^{\prime}(x) \\
& =\frac{1}{4^{n}}\left(D\left(2^{n-1}(x+e), 2^{n-1}(x-e)\right)-D\left(2^{n-1}(x+e), 2^{n-1}(e-x)\right)\right. \\
& \left.\quad+D\left(2^{n-1}(x-e), 2^{n-1}(e-x)\right)-D\left(2^{n-1}(x-e), 2^{n-1}(x-e)\right)\right)
\end{aligned}
$$

for all $x \in X$. This equality yields

$$
\begin{aligned}
& \|x H(e)-H(x)\| \\
& \begin{array}{l}
\leq \lim _{n \rightarrow \infty} \frac{1}{4^{n}}\left(\varphi\left(2^{n-1}(x+e), 2^{n-1}(x-e)\right)+\varphi\left(2^{n-1}(x+e), 2^{n-1}(e-x)\right)\right. \\
\left.\quad+\varphi\left(2^{n-1}(x-e), 2^{n-1}(e-x)\right)+\varphi\left(2^{n-1}(x-e), 2^{n-1}(x-e)\right)\right)
\end{array} \\
& \leq \lim _{n \rightarrow \infty} \frac{L^{n-1}}{4}\left(\begin{array}{r}
\varphi(x+e, x-e)+\varphi(x+e, e-x) \\
=
\end{array} \quad+\varphi(x-e, e-x)+\varphi(x-e, x-e)\right)
\end{aligned}
$$

for all $x \in X \backslash\{0\}$, i.e.,

$$
H(x)=x H(e)
$$

for all $x \in X \backslash\{0\}$. Since $H(0)=0$ and the last equality obviously holds for $x=0, H: X \rightarrow X$ is a $\mathbb{K}$-linear mapping. 
Finally, it follows from (6) and (11) that

$$
\begin{aligned}
& \|f(x)-g(0)-x h(x)\| \leq \varphi(x, 0) \\
& \|f(0)-g(x)+x h(x)\| \leq \varphi(0, x) \\
& \|f(0)-g(0)\| \leq \varphi(0,0) \\
& \|x h(x)-x H(x)-x h(0)\|=\|x\|\|h(x)-H(x)-h(0)\| \leq \frac{\Phi(x,\|x\| e)}{1-L}
\end{aligned}
$$

for all $x \in X \backslash\{-e, 0, e\}$. Using the above inequalities and (9), we easily obtain the inequalities (7) and (8).

Case 2. Suppose $\varphi$ satisfies (5) and we define a mapping $J: S \rightarrow S$ by

$$
J u(x):=2 u\left(\frac{x}{2}\right) \quad(x \in X)
$$

for all $u \in S$. Notice that the equality

$$
J^{n} u(x)=2^{n} u\left(\frac{x}{2^{n}}\right)
$$

holds for all $n \in \mathbb{N}_{0}$ and $x \in X$.

Assume that $u, v \in S$ and $K \in[0, \infty]$ is an arbitrary constant with $d(u, v) \leq K$. By definition of $d$ and (5), we have

$$
\|J u(x)-J v(x)\|=2\left\|u\left(\frac{x}{2}\right)-v\left(\frac{x}{2}\right)\right\| \leq 2 K \frac{\Phi\left(\frac{x}{2}, \frac{\|x\| e}{2}\right)}{\frac{\|x\|}{2}} \leq L K \frac{\Phi(x,\|x\| e)}{\|x\|}
$$

for all $x \in X \backslash\{-e, 0, e\}$, which again implies that

$$
d(J u, J v) \leq L d(u, v)
$$

for any $u, v \in S$. This means that $J$ is a strictly contractive self-mapping of $S$ with Lipschitz constant $L$.

Considering (3), we make a somewhat long and tedious calculation to get

$$
\begin{aligned}
h^{\prime}(x)-J h^{\prime}(x)=h(x)- & h(0)-2\left(h\left(\frac{x}{2}\right)-h(0)\right) \\
= & \frac{4}{\|x\|}\left(D\left(\frac{1}{4}(x+\|x\| e), \frac{1}{4}(x-\|x\| e)\right)\right. \\
& -D\left(\frac{1}{4}(x+\|x\| e), \frac{1}{4}(\|x\| e-x)\right) \\
& +D\left(\frac{1}{4}(x-\|x\| e), \frac{1}{4}(\|x\| e-x)\right) \\
& -D\left(\frac{1}{4}(x-\|x\| e), \frac{1}{4}(x-\|x\| e)\right) \\
& -\frac{1}{2} D\left(\frac{1}{2} x+\frac{1}{4}\|x\| e, \frac{1}{2} x-\frac{1}{4}\|x\| e\right) \\
& +\frac{1}{2} D\left(\frac{1}{2} x+\frac{1}{4}\|x\| e, \frac{1}{4}\|x\| e-\frac{1}{2} x\right) \\
& -\frac{1}{2} D\left(\frac{1}{2} x-\frac{1}{4}\|x\| e, \frac{1}{4}\|x\| e-\frac{1}{2} x\right) \\
& \left.+\frac{1}{2} D\left(\frac{1}{2} x-\frac{1}{4}\|x\| e, \frac{1}{2} x-\frac{1}{4}\|x\| e\right)\right)
\end{aligned}
$$


for all $x \in X \backslash\{-e, 0, e\}$. Using (5) and (6) and in view of (10), we notice that

$$
\left\|h^{\prime}(x)-J h^{\prime}(x)\right\|=\left\|h(x)-h(0)-2\left(h\left(\frac{x}{2}\right)-h(0)\right)\right\| \leq L \frac{\Phi(x,\|x\| e)}{\|x\|}
$$

for all $x \in X \backslash\{-e, 0, e\}$, which means that $d\left(h^{\prime}, J h^{\prime}\right) \leq L<\infty$.

In view of Theorem 2, the sequence $\left\{J^{n} h^{\prime}\right\}$ converges to a fixed point $H: X \rightarrow X$ of $J$ that is uniquely determined in the set $T=\left\{u \in S: d\left(h^{\prime}, u\right)<\infty\right\}$. We notice that

$$
d\left(h^{\prime}, H\right) \leq \frac{1}{1-L} d\left(h^{\prime}, J h^{\prime}\right) \leq \frac{L}{1-L},
$$

from which the second inequality in (9) follows.

We make a routine calculation by using (3) to obtain

$$
\begin{aligned}
x J^{n} h^{\prime}(e)-J^{n} h^{\prime}(x)=4^{n}( & D\left(\frac{x+e}{2^{n+1}}, \frac{x-e}{2^{n+1}}\right)-D\left(\frac{x+e}{2^{n+1}}, \frac{e-x}{2^{n+1}}\right) \\
& \left.+D\left(\frac{x-e}{2^{n+1}}, \frac{e-x}{2^{n+1}}\right)-D\left(\frac{x-e}{2^{n+1}}, \frac{x-e}{2^{n+1}}\right)\right)
\end{aligned}
$$

for all $x, y \in X$. This equality together with (5) yields

$$
\begin{array}{r}
\|x H(e)-H(x)\| \leq \lim _{n \rightarrow \infty} 4^{n}\left(\varphi\left(\frac{x+e}{2^{n+1}}, \frac{x-e}{2^{n+1}}\right)+\varphi\left(\frac{x+e}{2^{n+1}}, \frac{e-x}{2^{n+1}}\right)\right. \\
\left.+\varphi\left(\frac{x-e}{2^{n+1}}, \frac{e-x}{2^{n+1}}\right)+\varphi\left(\frac{x-e}{2^{n+1}}, \frac{x-e}{2^{n+1}}\right)\right) \\
\leq \lim _{n \rightarrow \infty} \frac{L^{n+1}}{4}(\varphi(x+e, x-e)+\varphi(x+e, e-x) \\
=0
\end{array}
$$

for all $x \in X \backslash\{0\}$, i.e.,

$$
H(x)=x H(e)
$$

for all $x \in X \backslash\{0\}$. Hence, we conclude that $H: X \rightarrow X$ is a $\mathbb{K}$-linear mapping.

Finally, by (6) and (12), we get

$$
\begin{aligned}
& \|f(x)-g(0)-x h(x)\| \leq \varphi(x, 0) \\
& \|f(0)-g(x)+x h(x)\| \leq \varphi(0, x), \\
& \|f(0)-g(0)\| \leq \varphi(0,0) \\
& \|x h(x)-x H(x)-x h(0)\|=\|x\|\|h(x)-H(x)-h(0)\| \leq \frac{L \Phi(x,\|x\| e)}{1-L}
\end{aligned}
$$

for all $x \in X \backslash\{-e, 0, e\}$. Using the above inequalities and (9), we easily obtain the inequalities (7) and (8). 


\section{Applications}

In the following corollary, we set $\varphi(x, y):=\theta\left(\|x\|^{p}+\|y\|^{p}\right)$ and $L:=\frac{2^{p}}{4}$, where $p$ and $\theta$ are positive real constants with $0<p<2$. Then, $\varphi$ satisfies (4) and $0<L<1$. Further, if we use the following notation

$$
K(x):=2\|x+\| x\|e\|^{p}+6\|x-\| x\|e\|^{p}+\|2 x+\| x\|e\|^{p}+3\|2 x-\| x\|e\|^{p},
$$

then it follows from (10) that $\Phi(x,\|x\| e)=\frac{\theta}{2^{p}} K(x)$ for all $x \in X \backslash\{-e, 0, e\}$.

Corollary 1. Suppose $X$ is a commutative unital Banach algebra over $\mathbb{K}$ with a unit element e (or $X$ is a normed field of characteristic different from 2). Let $p$ and $\theta$ be positive real numbers with $0<p<2$. If functions $f, g, h: X \rightarrow X$ satisfy the inequality

$$
\|f(x)-g(y)-(x-y) h(x+y)\| \leq \theta\left(\|x\|^{p}+\|y\|^{p}\right)
$$

for all $x, y \in X$, then there exist $a, b, c \in X$ such that

$$
\begin{aligned}
& \left\|f(x)-a x^{2}-b x-c\right\| \leq \theta\|x\|^{p}+\frac{4 \theta K(x)}{2^{p}\left(4-2^{p}\right)} \\
& \left\|g(x)-a x^{2}-b x-c\right\| \leq \theta\|x\|^{p}+\frac{4 \theta K(x)}{2^{p}\left(4-2^{p}\right)} \\
& \|h(x)-a x-h(0)\| \leq \frac{4 \theta K(x)}{2^{p}\left(4-2^{p}\right)\|x\|}
\end{aligned}
$$

for all $x \in X \backslash\{-e, 0, e\}$. In particular, if $0<p<2$ but $p \neq 1$, then the $a, b, c \in X$ are determined uniquely.

Proof. If we put $\varphi(x, y):=\theta\left(\|x\|^{p}+\|y\|^{p}\right)$ and $L:=\frac{2^{p}}{4}$, then $\varphi$ satisfies (4). Further, we put $a:=H(e)$, $b:=h(0)$ and $c:=f(0)=g(0)$ in Theorem 3. Finally, assume that $0<p<2$ but $p \neq 1$ and there exist $a^{\prime}, b^{\prime} \in X$ such that

$$
\left\|h(x)-a^{\prime} x-b^{\prime}\right\| \leq \frac{4 \theta K(x)}{2^{p}\left(4-2^{p}\right)\|x\|} .
$$

Then, by the third inequality of (13), we get

$$
\left\|\left(a-a^{\prime}\right) x+b-b^{\prime}\right\| \leq \frac{8 \theta K(x)}{2^{p}\left(4-2^{p}\right)\|x\|}
$$

for all $x \in X \backslash\{-e, 0, e\}$. If we substitute $2^{n} x$ for $x$ in the last inequality and divide the resulting inequality by $2^{n}$, then we have

$$
\left\|\left(a-a^{\prime}\right) x+\frac{b-b^{\prime}}{2^{n}}\right\| \leq \frac{8 \theta K(x)}{2^{p}\left(4-2^{p}\right)\|x\|}\left(\frac{2^{p}}{4}\right)^{n} \rightarrow 0 \text { as } n \rightarrow \infty
$$

for each $x \in X \backslash\{-e, 0, e\}$, i.e., $a=a^{\prime}$. To prove the uniqueness of $b$, we substitute $2^{n} x$ when $0<p<1$ and $\frac{1}{2^{n}} x$ when $1<p<2$. Similarly, we prove the uniqueness of $c$.

We now set $\varphi(x, y):=\theta\left(\|x\|^{p}+\|y\|^{p}\right)$ and $L:=\frac{4}{2^{p}}$, where $p$ and $\theta$ are positive real constants with $p>2$. Then, $\varphi$ satisfies (5) and $0<L<1$. Further, it follows from (10) that $\Phi(x,\|x\| e)=\frac{\theta}{2^{p}} K(x)$ for all $x \in X \backslash\{-e, 0, e\}$. 
Corollary 2. Suppose $X$ is a commutative unital Banach algebra over $\mathbb{K}$ with a unit element $e$ (or $X$ is a normed field of characteristic different from 2 ). Let $p$ and $\theta$ be positive real numbers with $p>2$. If functions $f, g, h: X \rightarrow X$ satisfy the inequality

$$
\|f(x)-g(y)-(x-y) h(x+y)\| \leq \theta\left(\|x\|^{p}+\|y\|^{p}\right)
$$

for all $x, y \in X$, then there exist unique $a, b, c \in X$ such that

$$
\begin{aligned}
& \left\|f(x)-a x^{2}-b x-c\right\| \leq \theta\|x\|^{p}+\frac{4 \theta K(x)}{2^{p}\left(2^{p}-4\right)} \\
& \left\|g(x)-a x^{2}-b x-c\right\| \leq \theta\|x\|^{p}+\frac{4 \theta K(x)}{2^{p}\left(2^{p}-4\right)} \\
& \|h(x)-a x-h(0)\| \leq \frac{4 \theta K(x)}{2^{p}\left(2^{p}-4\right)\|x\|}
\end{aligned}
$$

for all $x \in X \backslash\{-e, 0, e\}$.

Proof. If we put $\varphi(x, y):=\theta\left(\|x\|^{p}+\|y\|^{p}\right)$ and $L:=\frac{4}{2^{p}}$, then $\varphi$ satisfies (5). Further, we put $a:=H(e)$, $b:=h(0)$ and $c:=f(0)=g(0)$ in Theorem 3. In addition, we may prove the uniqueness of $a, b$ and $c$ by using a similar argument as in the proof of Corollary 1.

Finally, we set $\varphi(x, y):=\delta$ and $L:=\frac{1}{4}$, where $\delta$ is a positive real constant. Then, $\varphi$ satisfies (4) and $\Phi(x,\|x\| e)=6 \delta$ for all $x \in X \backslash\{-e, 0, e\}$.

Corollary 3. Suppose $X$ is a commutative unital Banach algebra over $\mathbb{K}$ with a unit element e (or $X$ is a normed field of characteristic different from 2 ). Let $\varepsilon$ be a positive real number. If functions $f, g, h: X \rightarrow X$ satisfy the inequality

$$
\|f(x)-g(y)-(x-y) h(x+y)\| \leq \varepsilon
$$

for all $x, y \in X$, then there exist unique $a, b \in X$ such that

$$
\begin{aligned}
& \left\|f(x)-a x^{2}-b x-f(0)\right\| \leq 10 \varepsilon \\
& \left\|g(x)-a x^{2}-b x-g(0)\right\| \leq 10 \varepsilon \\
& \|h(x)-a x-h(0)\| \leq \frac{8 \varepsilon}{\|x\|}
\end{aligned}
$$

for all $x \in X \backslash\{-e, 0, e\}$.

Proof. If we put $\varphi(x, y):=\varepsilon$ and $L:=\frac{1}{4}$, then $\varphi$ satisfies (4) and $\Phi(x,\|x\| e)=6 \varepsilon$. Further, we put $a:=H(e), b:=h(0)$ and $c:=f(0)=g(0)$ in Theorem 3. Finally, we prove the uniqueness of $a$ and $b$ in a similar way to the proof of Corollary 1.

Acknowledgments: Soon-Mo Jung was supported by Basic Science Research Program through the National Research Foundation of Korea (NRF) funded by the Ministry of Education (No. 2016R1D1A1B03931061).

Author Contributions: Soon-Mo Jung and Yang-Hi Lee conceived and wrote the paper.

Conflicts of Interest: The authors declare no conflict of interest.

\section{References}

1. Ulam, S.M. Problems in Modern Mathematics; Wiley: New York, NY, USA, 1960; Chapter VI.

2. Hyers, D.H. On the stability of the linear functional equation. Proc. Natl. Acad. Sci. USA 1941, 27, $222-224$.

3. Aoki, T. On the stability of the linear transformation in Banach spaces. J. Math. Soc. Jpn. 1950, 2, 64-66. 
4. Găvruța, P. A generalization of the Hyers-Ulam-Rassias stability of approximately additive mappings. J. Math. Anal. Appl. 1994, 184, 431-436.

5. Rassias, T.M. On the stability of the linear mapping in Banach spaces. Proc. Am. Math. Soc. 1978, 72, $297-300$.

6. Forti, G.L. Hyers-Ulam stability of functional equations in several variables. Aequ. Math. 1995, 50, $143-190$.

7. Hyers, D.H.; Rassias, T.M. Approximate homomorphisms. Aequ. Math. 1992, 44, 125-153.

8. Jung, S.-M. Hyers-Ulam-Rassias Stability of Functional Equations in Nonlinear Analysis; Springer: New York, NY, USA, 2011.

9. Jung, S.-M.; Sahoo, P.K. On the stability of a mean value type functional equation. Demonstr. Math. 2000, 33, 793-796.

10. Aczél, J. A mean value property of the derivative of quadratic polynomials-Without mean values and derivatives. Math. Mag. 1985, 58, 42-45.

11. Haruki, S. A property of quadratic polynomials. Am. Math. Mon. 1979, 86, 577-579.

12. Kannappan, P.L.; Sahoo, P.K.; Jacobson, M.S. A characterization of low degree polynomials. Demonstr. Math. 1995, 28, 87-96.

13. Sahoo, P.K.; Riedel, T. Mean Value Theorems and Functional Equations; World Scientific: Singapore; Hackensack, NJ, USA; London, UK; Hong Kong, China, 1998.

14. Cădariu, L.; Radu, V. Fixed points and the stability of Jensen's functional equation. J. Inequal. Pure Appl. Math. 2003, 4, 4 .

15. Cădariu, L.; Radu, V. Fixed points and the stability of quadratic functional equations. An. Univ. Timisoara Ser. Mat.-Inform. 2003, 41, 25-48.

16. Cădariu, L.; Radu, V. On the Stability of the Cauchy Functional Equation: A Fixed Point Approach in Iteration Theory; Grazer Mathematische Berichte; Karl-Franzens-Universitäet: Graz, Austria, 2004; Volume 346, pp. 43-52.

17. Choi, G.; Jung, S.-M.; Lee, Y.-H. Approximation properties of solutions of a mean value type functional inequalities. J. Nonlinear Sci. Appl. 2017, 10, 4507-4514.

18. Shehu, Y.; Cai, G.; Iyiola, O.S. Iterative approximation of solutions for proximal split feasibility problems. Fixed Point Theory Appl. 2015, 2015, 123.

19. Diaz, J.B.; Margolis, B. A fixed point theorem of the alternative for contractions on a generalized complete metric space. Bull. Am. Math. Soc. 1968, 74, 305-309.

20. Rus, I.A. Principles and Applications of Fixed Point Theory; Dacia: Cluj-Napoca, Romania, 1979. (In Romanian)

21. Rudin, W. Functional Analysis, 2nd ed.; McGraw-Hill: New York, NY, USA, 1991.

(C) 2017 by the authors. Licensee MDPI, Basel, Switzerland. This article is an open access article distributed under the terms and conditions of the Creative Commons Attribution (CC BY) license (http://creativecommons.org/licenses/by/4.0/). 